\title{
Comparison of fluorescence in-situ hybridisation with dual-colour in-situ hybridisation for assessment of HER2 gene amplification of breast cancer in Hong Kong
}

\author{
Scott MC Tang, Inda S Soong, MY Luk, Dacita TK Suen, F Hioe, Ellen PS Man, Obe KL Tsun, US Khoo *
}

\section{A B S T R A C T}

Objectives: To compare the PathVysion fluorescence in-situ hybridisation assay with the INFORM HER2 Dual in-situ hybridisation assay on 104 invasive breast cancers with a broad spectrum of immunohistochemistry scores.

Methods: This case series involved consecutive patients diagnosed with invasive breast carcinoma with equivocal immunohistochemistry score and referred for further HER2 assessment from the departments of Surgery and/or Clinical Oncology of the two hospitals between January 2013 and February 2014. An additional 10 cases with negative HER2 immunohistochemistry and 11 cases with positive HER2 immunohistochemistry were further included.

Results: The results of both fluorescence in-situ hybridisation and dual in-situ hybridisation were available in 99 of 104 cases, respectively. Student's $t$ test showed no statistically significant difference in the mean number of HER2 count, CEP17 copies, or HER2/CEP17 ratio between that obtained by fluorescence in-situ hybridisation and that obtained by dual in-situ hybridisation. Pearson's correlation of results for the two assays was strong for HER2/CEP17 signal ratio $(\mathrm{R}=0.963, \mathrm{P}<0.001)$ and mean HER2 copies per nucleus $(\mathrm{R}=0.897$, $\mathrm{P}<0.001$ ). Overall agreement was $96.0 \%$ (95 out

This article was published on 29 Jan 2016 at www.hkmj.org. of 99 cases, $\mathrm{k}=0.882$ ). Three of the four discordant cases were equivocal for either fluorescence in-situ hybridisation or dual in-situ hybridisation. The results of immunohistochemistry $0 / 1+$ and $3+$ cases showed $100 \%$ concordance between the two assays. The failure rate was $0.96 \%$ for fluorescence in-situ hybridisation and $3.85 \%$ for dual in-situ hybridisation. Cases that failed for fluorescence in-situ hybridisation were successful for dual in-situ hybridisation and vice versa.

Conclusions: Our study showed that dual in-situ hybridisation is a reliable and useful option for HER2 testing in breast cancer.

\section{Hong Kong Med J 2016;22:144-51}

DOI: $10.12809 / \mathrm{hkmj} 144458$
SMC Tang, MB, ChB, MRCSEd
${ }^{2}$ IS Soong, FRCR, FHKAM (Radiology)
${ }^{3}$ MY Luk, FRCR, FHKAM (Radiology)
${ }^{4}$ DTK Suen, FRACS, FHKAM (Surgery)
${ }^{5} \mathrm{~F}$ Hioe, FHKCPath, FHKAM (Pathology)
${ }^{1}$ EPS Man, MMedSc
OKL Tsun, CFIAC, MMedSc
${ }^{1}$ US Khoo *, FRCPath, FHKAM (Pathology)
Department of Pathology, LKS Faculty of Medicine, The University of Hong Kong, Hong Kong
Department of Clinical Oncology, Pamela Youde Nethersole Eastern Hospital, Chai Wan, Hong Kong
Department of Clinical Oncology, Queen Mary Hospital, Pokfulam, Hong Kong
Department of Surgery, Queen Mary Hospital, Pokfulam, Hong Kong
Chai Wan, Hong Kong
${ }^{5}$ Department of Pathology, Pamela Youde Nethersole Eastern Hospital,

*Corresponding author: uskhoo@hku.hk

New knowledge added by this study

Our local experience confirmed the diagnostic value of dual in-situ hybridisation (DISH) for assessment of HER2 gene amplification in breast cancer, with excellent correlation between fluorescence in-situ hybridisation assay (FISH) and DISH results. Cases that failed FISH were successful with DISH and vice versa.

Implications for clinical practice or policy

DISH provides a reliable and useful option for HER2 testing in breast cancer, and offers some practical advantages.

\section{Introduction}

Breast cancer is the most common female malignancy. In Hong Kong, breast cancer accounted for about $26 \%$ of newly diagnosed cancers and $10 \%$ of cancer mortality in women. ${ }^{1}$ The human epidermal growth factor receptor type 2 (HER2) gene is a very important predictor of clinical outcome in breast cancer patients; protein overexpression or gene amplification is associated with higher rates of recurrence and higher mortality, ${ }^{2}$ and responsiveness 
to endocrine ${ }^{3}$ and chemotherapeutic regimens. ${ }^{4}$ Trastuzumab (Herceptin; Genentech Inc, South San Francisco, US) that targets the HER2 oncoprotein is an established therapy for HER2-positive breast cancer patients in both the adjuvant $\mathrm{t}^{5,6}$ and metastatic settings. ${ }^{7,8}$ Thus HER2 status is an important guide to the use of systemic adjuvant therapies. Because of the expense and potential life-threatening cardiotoxicity of Herceptin therapy, accuracy of the HER2 testing is of primary importance.

The American Society of Clinical Oncology and the College of American Pathologists (ASCO/ CAP) have issued guidelines recommending determination of HER2 status in all patients with invasive breast cancer (early stage, or recurrence/ metastasis) to guide therapy. ${ }^{9-11}$ Following the guidelines published in 2007, many laboratories now use immunohistochemistry (IHC) as a screening test, with fluorescence in-situ hybridisation (FISH) used to determine HER2 status in equivocal IHC cases and to serve as a reference standard. The prevalence of HER2 gene amplification in breast cancer varies between studies, ranging from about $20 \%$ to $30 \% .^{10,12-15}$

Although FISH remains the 'gold standard' to determine HER2 gene amplification, in 2013, the INFORM HER2 Dual-ISH DNA Probe Cocktail assay (Ventana Medical Systems, Tucson, US) was approved by the Food and Drug Administration (FDA) for determination of HER2 gene amplification status as an alternative to FISH. ${ }^{16}$ It utilises silver in-situ hybridisation (ISH) to detect the HER2 gene and chromogenic ISH for the chromosome 17 centromere (CEP17) for visualisation on the same slide under light microscopy. Both FISH and dual-colour in-situ hybridisation (DISH) use formalin-fixed, paraffin-embedded breast cancer tissue specimens, but DISH has the advantage of allowing light microscopy assessment. This enables concurrent visualisation of histomorphological features with HER2 gene status, permitting the invasive component of the tumour to be more easily identified and analysed. Unlike FISH where the immunofluorescent signals will fade, DISH specimens can be archived and retrieved indefinitely. The assay can be processed on an automated platform and can contribute to reduced reporting turnaround time.

Some studies that compared FISH and DISH assays have shown excellent concordance. ${ }^{17-20}$ We have previously reported the prevalence and concordance between IHC HER2 overexpression and ISH assay of breast cancers in Hong Kong. ${ }^{21}$ Funded by the SK Yee Medical Foundation to provide HER2 FISH testing in patients receiving treatment from public hospitals, and with subsequent FDA approval to provide the alternative HER2 DISH test, we performed a validation study in our laboratory

\section{熒光原位雜交技術與雙色原位雜交技術檢測香港} 乳癌HER2狀態的比較

\author{
鄧文俊、宋崧、陸美儀、孫杜琪、丘斐、萬佩心、秦家麗 \\ 邱瑋璇
}

目的：在 104 個涵蓋不同免疫組織化學分析評分結果的侵犯性乳癌 個案樣本上比較PathVysion焱光原位雜交技術 (FISH) 和INFORM HER2雙色原位雜交技術（DISH）

方法：這項病例系列研究納入 2013年1月至2014年2月期間, 所有免 疫組織化學結果顯示模棱兩可的侵犯性乳癌個案樣本而被轉介至香港 兩所公立醫院的病理學、外科和臨床腫瘤科部門作進一步HER2基因 評估的個案樣本，以及 10 個陰性免疫組織化學分析結果和 11 個陽性免 疫組織化學分析結果的樣本。

結果：104個樣本當中, 有99個成功取得FISH和DISH結果作比較。 使用兩個方法得出的平均HER2 數目、CEP17 標記數量和HER2/ $\mathrm{CEP} 17$ 比例並沒有顯著差異。HER2/CEP17 比例（ $\mathrm{R}=0.963$ ， $\mathrm{P}<0.001)$ 和每個細胞核的平均 HER2 數目的Pearson相關系數 $(\mathrm{R}=0.897, \mathrm{P}<0.001)$ 均呈強線性關係。兩項技術的結果整體一致 率為96.0\%（99個樣本中有95個結果相同， $\mathrm{K}=0.882$ )。在測試結果不 一致的 4 個樣本當中, 有 3 個在FISH或DISH其中一種技術得出模梭兩 可的結果。免疫組織化學結果顯示 $0 / 1+$ 和 $3+$ 的樣本利用這兩種技術的 化驗結果完全一致。不能成功進行FISH及DISH檢測的失敗率分別是 $0.96 \%$ 及 $3.85 \%$ 。所有不能進行FISH檢測的個案皆可成功通過DISH檢 測並獲得結果, 反之亦然。

結論：DISH是偵測乳癌HER2一個可靠和有用的測試方法。

to compare the results of FISH and DISH tests in determining HER2 status in breast cancer, before offering DISH for routine testing.

\section{Methods}

\section{Patients}

This retrospective study included 104 breast cancer cases referred from the Department of Clinical Oncology of Pamela Youde Nethersole Eastern Hospital and Queen Mary Hospital, and from the Department of Surgery, Queen Mary Hospital. Case selection was based on IHC results representing three IHC categories: negative (0 or $1+$ HER2 score), equivocal (2+ HER2 score), and positive (3+ HER2 score) for HER2 overexpression, interpreted and classified according to the ASCO/CAP guidelines at the time of presentation. Slides from both hospitals were reviewed and confirmed to fulfil the updated classification score of the ASCO/CAP 2013 guidelines. These included 83 consecutive cases between January 2013 and February 2014 that were equivocal for HER2 IHC (2+ score). In addition, 10 cases that were reported to be HER2 IHC-negative ( 0 or $1+$ score) and 11 cases reported as HER2 IHCpositive ( $3+$ score) were added to the study. 
All patients had undergone surgery for invasive breast cancer. None had received preoperative chemotherapy. All tests were performed at the CAPaccredited University Pathology Laboratory of the University of Hong Kong.

Serial 4-6 $\mu \mathrm{m}$ sections were prepared from formalin-fixed, paraffin-embedded tumour tissue. Sections were sent for haematoxylin and eosin (H\&E) and immunohistochemical staining. The H\&E sections were reviewed by a certified pathologist. Areas of invasive tumour were marked on the slide for assessment. For FISH analysis, only the invasive tumour components were included for assessment, being mindful that it is difficult to distinguish in-situ from invasive carcinoma under assessment by dark field imaging.

\section{Fluorescence in-situ hybridisation analysis}

The FISH testing was performed using the FDAapproved PathVysion HER2 DNA Probe Kit (Abbott Molecular Inc, Illinois, US). All samples were processed following previously defined protocols in compliance with the manufacturer's instructions. Briefly, the slides were baked overnight at $56^{\circ} \mathrm{C}$, deparaffinised, dehydrated, and air-dried. This was followed by protease treatment for 30 minutes. DNA was denatured at $72^{\circ} \mathrm{C}$ and hybridisation carried out at $37^{\circ} \mathrm{C}$ for 16 hours.

Slides were then washed and air-dried. Counterstain was applied and the slide covered and sealed. Positive and negative controls were included for each batch of analysis. Slides were then visualised under a fluorescence microscope (CGH workstation,
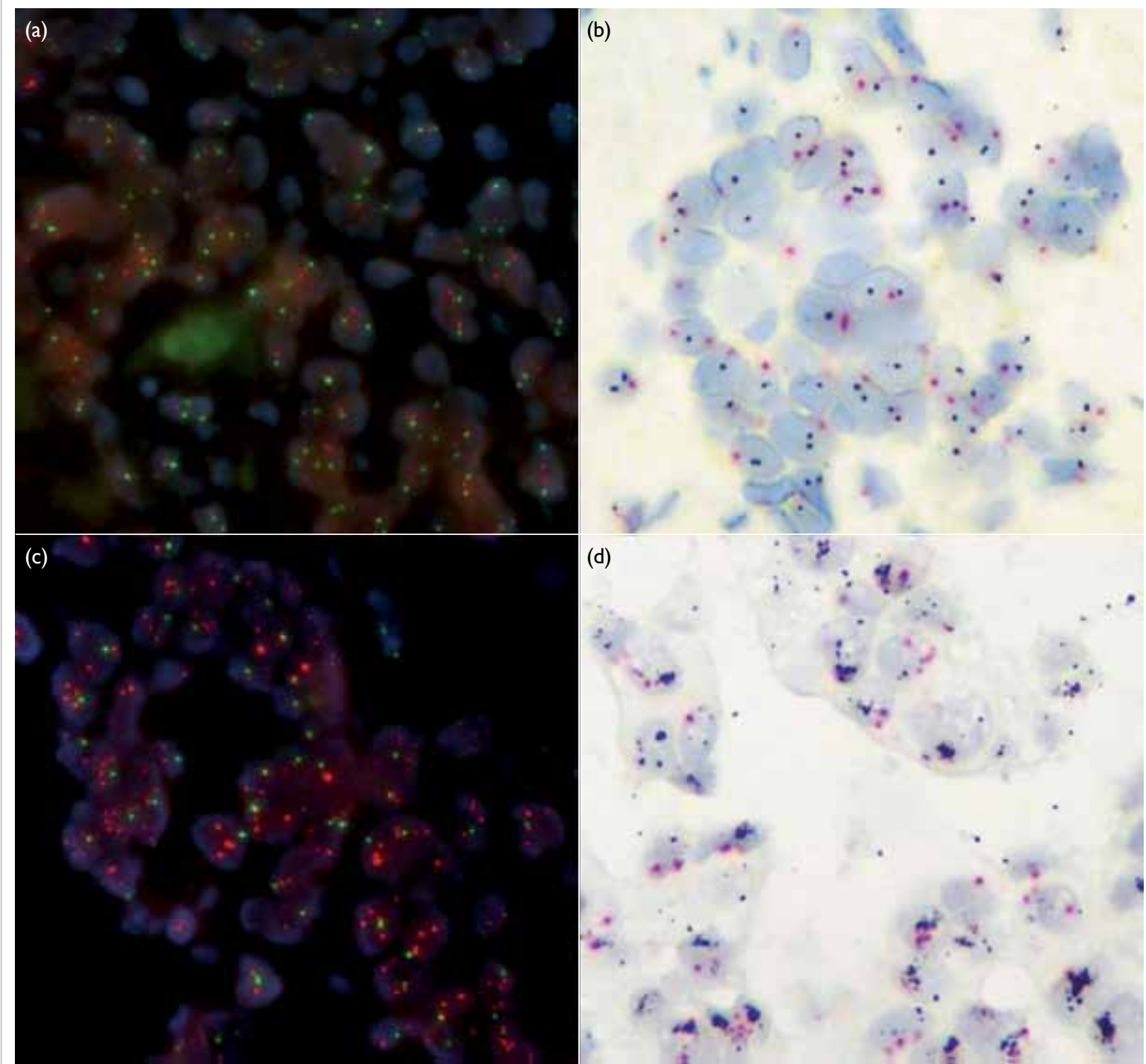

FIG I. FISH and DISH results of two representative cases

(a) FISH result of case \#34 with a signal ratio of I.22 (non-amplified). (b) DISH result of the same patient with a signal ratio of I.45 (non-amplified). (c) FISH result of case \# I with a signal ratio of 4.4 (amplified). (d) DISH result of the same patient with a signal ratio of 4.97 (amplified)

Abbreviations: DISH = dual-colour in-situ hybridisation; FISH = fluorescence in-situ hybridisation 
Leica Q550CW) with a 100x objective using a triple filter that included DAPI, GFP, and Texas Red. The HER 2 gene is visualised as a red/orange signal, and the CEP17 as a green signal.

The number of HER2 and CEP17 signals was counted for 20 nuclei. The signal ratio was then calculated for each case. One to three photos were taken for each case. Following criteria given by the ASCO/CAP guidelines, a FISH result was rejected and repeated if: controls were not as expected; observer could not find and count at least two areas of invasive tumour; $>25 \%$ of signals were unscorable due to weak signals; $>10 \%$ of signals occurred over cytoplasm; nuclear resolution was poor; or autofluorescence was strong. ${ }^{9}$ Figures $1 \mathrm{a}$ and $1 \mathrm{c}$ show representative FISH results of a sample from two patients.

\section{Dual-colour in-situ hybridisation analysis}

The DISH testing was performed using the INFORM HER2 Dual-ISH DNA Probe Cocktail assay (Ventana Medical Systems, Tucson, US). All samples were processed automatically by BenchMark XT (Ventana Medical Systems). The HER2 was detected by a dinitrophenyl (DNP)-labelled probe and visualised in black colour utilising the ultraView Silver ISH DNP Detection Kit (Ventana Medical Systems). The CEP17 was targeted with a digoxigenin (DIG)-labelled probe and detected as a red signal using the ultraView Red ISH DIG Detection Kit (Ventana Medical Systems). Haematoxylin II was used as counterstain. Positive and negative controls were included for each batch of analysis. Slides were visualised under a 40x objective with a light microscope. Signal counting was performed according to the manufacturer's interpretation guide. The number of HER2 and CEP17 signals was counted for 20 nuclei and the signal ratio calculated for each case. One to three photos were taken for each slide. A DISH result was rejected and repeated according to the same criteria as FISH in the ASCO/ CAP guidelines. Figures $1 \mathrm{~b}$ and $1 \mathrm{~d}$ show the DISH results for the same two patients in Figures 1a and 1c.

\section{Scoring criteria}

For signal counting of FISH and DISH, the number of HER2 gene signals and CEP17 signals were counted in 20 tumour nuclei. The HER2/CEP17 signal ratio and mean number of HER2 signals per nucleus was calculated. HER2 gene amplification status was then determined according to ASCO/ CAP 2013 guidelines for dual-probe ISH assay. ${ }^{9}$ For cases that presented before the 2013 guidelines, raw data of signal enumeration were retrieved, and the results reclassified after applying the new guideline. Briefly, if HER2/CEP17 ratio was $\geq 2.0$, it was classified as positive. If HER2/CEP17 ratio was <2.0, classification would be based on mean HER2 copy number per nucleus. If mean HER2 copy number per nucleus was $\geq 6$, then it was positive; if it was $\geq 4.0$ and $<6.0$, then it was equivocal; if it was $<4.0$, then it was negative.

\section{Statistical analyses}

Cases that failed FISH or DISH analysis were excluded from statistical analysis. The Statistical Package for the Social Sciences (Windows version 20.0; SPSS Inc, Chicago [IL], US) was used. The following statistical analyses were performed:

(1) First, one-way analysis of variance (ANOVA) test was used to analyse the relationship between IHC result and quantitative results of FISH and DISH.

(2) We tested whether DISH underestimated or overestimated the number of HER2 or CEP17 copies when compared with FISH. The null hypothesis was that there was no difference. Student's $t$ test was used to examine the result of both tests on mean HER2/CEP17 ratio and mean HER2 copy number per nucleus. A $P$ value of $<0.05$ indicated a statistically significant difference.

(3) We also used the Bland-Altman plots to show the degree of agreement graphically. Linear regression was used to show the relationship of FISH and DISH results. Pearson productmoment correlation coefficient (R) was calculated to evaluate the correlation between quantitative results of FISH and DISH. A positive $\mathrm{R}$ ( 0 to 1 ) indicates positive correlation and a negative $\mathrm{R}$ indicates negative correlation. If $-1 \leq R<-0.7$ or $0.7<R \leq 1$, it indicates strong association. A P value of $<0.05$ indicated a statistically significant difference.

(4) To evaluate agreement between FISH and DISH in the classification of HER2 gene amplification status, Cohen's Kappa coefficient was used to factor in the possibility that the two tests agreed due to chance. We also calculated simple agreement percentage for comparison with results of other studies.

All tests were two-sided. All confidence intervals (CIs) and $\mathrm{P}$ values were included in the results.

\section{Results}

\section{Failure cases}

Both FISH and DISH results were available in 99 of 104 cases. One case (\#85) failed FISH analysis after two attempts. Four cases (\#14, \#78, \#84, \#101) failed DISH analysis after two attempts. The failure rate was $0.96 \%$ for FISH and 3.85\% for DISH. The reasons for failure included criteria for result rejection as stated in ASCO/CAP guidelines. 
TABLE I. ANOVA analysis of IHC and ISH results

\begin{tabular}{lcccc}
\hline \multirow{2}{*}{ FISH and DISH results } & \multicolumn{3}{c}{ IHC results (mean \pm SD) } & \multirow{2}{*}{ P value } \\
\cline { 2 - 4 } & $\mathbf{0 / 1 +}$ & $\mathbf{2 +}$ & $\mathbf{3 +}$ & \\
\hline FISH & & & & \\
\hline HER2/CEP17 ratio & $1.29 \pm 0.10$ & $1.83 \pm 1.83$ & $5.12 \pm 2.45$ & $<0.001$ \\
\hline HER2 copies per nucleus & $1.98 \pm 0.39$ & $3.02 \pm 2.26$ & $8.03 \pm 3.46$ & $<0.001$ \\
\hline DISH & & & & \\
\hline HER2/CEP17 ratio & $1.42 \pm 0.28$ & $1.83 \pm 1.64$ & $4.73 \pm 2.22$ & $<0.001$ \\
\hline HER2 copies per nucleus & $2.46 \pm 0.43$ & $3.34 \pm 2.36$ & $8.85 \pm 4.14$ & $<0.001$ \\
\hline
\end{tabular}

Abbreviations: ANOVA = analysis of variance; DISH = dual-colour in-situ hybridisation; $\mathrm{FISH}=$ fluorescence in-situ hybridisation; $I \mathrm{HC}=$ immunohistochemistry; ISH = in-situ hybridisation; SD = standard deviation

TABLE 2. Comparison of HER2 and CEPI7 counts and HER2/CEPI7 ratio by FISH and DISH

\begin{tabular}{lccc}
\hline Counts/ratio & \multicolumn{2}{c}{ Mean \pm SD (range) } & \multirow{2}{*}{ P value } \\
\cline { 2 - 3 } & FISH & DISH & \\
\hline HER2 counts (20 cells) & $69.2 \pm 56.1(25-333)$ & $76.0 \pm 59.8(28-277)$ & N/A \\
HER2 counts per nucleus & $3.5 \pm 2.8(1.3-16.7)$ & $3.8 \pm 3.0(1.4-13.9)$ & 0.41 \\
CEP17 counts (20 cells) & $34.5 \pm 9.8(20-68)$ & $38.3 \pm 13.1(20-102)$ & N/A \\
HER2/CEP17 ratio & $2.1 \pm 2.1(1.1-14.5)$ & $2.1 \pm 1.8(1.05-11.0)$ & 0.86 \\
\hline
\end{tabular}

Abbreviations: DISH = dual-colour in-situ hybridisation; FISH = fluorescence in-situ hybridisation; N/A = not applicable; SD = standard deviation

TABLE 3. Comparison of amplification status results by FISH and DISH*

\begin{tabular}{lcccc}
\hline \multirow{2}{*}{ FISH } & \multicolumn{3}{c}{ DISH } & Total \\
\cline { 2 - 4 } & Amplified & Equivocal & Non-amplified & \\
\hline Amplified & 18 & 0 & 0 & 18 \\
Equivocal & 0 & 1 & 1 (Case \#8) & 2 \\
Non-amplified & 1 (Case \#83) & 2 (Cases \#13, 26) & 76 & 79 \\
Total & $\mathbf{1 9}$ & $\mathbf{3}$ & $\mathbf{7 7}$ & $\mathbf{9 9}$ \\
\hline
\end{tabular}

Abbreviations: DISH = dual-colour in-situ hybridisation; FISH = fluorescence in-situ hybridisation

* Overall agreement $=96.0 \%$ (95 out of 99 cases), and Cohen's Kappa coefficient was 0.882 (95\% confidence interval, $0.77-0.99, \mathrm{P}<0.00 \mathrm{I}$ )

\section{One-way analysis of variance}

The results of one-way ANOVA analysis are shown in Table 1 . For FISH versus IHC, the P value was $<0.001$ for mean HER2/CEP17 ratio and mean HER2 copies per nucleus. Both were $<0.05$, indicating a significantly different FISH reading for the different IHC groups. For DISH versus IHC, the P value was $<0.001$ for mean HER2/CEP17 ratio and mean HER2 copies per nucleus. Both were $<0.05$, indicating a significantly different DISH reading for the different IHC groups.

\section{Student's $\boldsymbol{t}$ test}

The result of Student's $t$ test is shown in Table 2. The mean number ( \pm standard deviation) of HER2 counts by FISH analysis was $3.5 \pm 2.8$, result by DISH was $3.8 \pm 3.0$ with no statistically significant difference between the results $(\mathrm{P}=0.41)$. The mean HER2/CEP17 ratio by FISH was $2.1 \pm 2.1$, result by DISH was $2.1 \pm 1.8$. There was no statistically significant difference between the results $(\mathrm{P}=0.86)$.

\section{Bland-Altman (limits of agreement) plot}

The Bland-Altman plot is shown in Figure 2a. For HER2 counts per nucleus, the mean difference (FISH-DISH) was 0.386 . The $95 \% \mathrm{CI}$ was -2.99 to 2.22. For HER2/CEP17 ratio, the mean difference (FISH-DISH) was 0.279 . The $95 \% \mathrm{CI}$ was -0.87 to 1.43.

\section{Linear regression and Pearson's correlation between the two in-situ hybridisation assays}

Scatter diagrams of DISH plotted against FISH results are shown in Figure 2b. Linear regression showed that DISH resulted in a lower HER2/CEP17 ratio than FISH, the tendency being more obvious at a higher ratio. Pearson's correlation coefficient was 0.897 (95\% CI, 0.84-0.95, $\mathrm{P}<0.001$ ) for mean HER2 copies per nucleus and 0.963 (95\% CI, 0.95-0.98, $\mathrm{P}<0.001)$ for HER2/CEP17 ratio. This indicated the correlation was excellent.

\section{Kappa's agreement between amplification} status results by the two in-situ hybridisation assays

The result of amplification status by DISH and FISH is shown in Table 3. Overall agreement of FISH and DISH was $96.0 \%$ (95 out of 99 cases), and Cohen's Kappa coefficient was 0.882 (95\% CI, 0.77-0.99, $\mathrm{P}<0.001)$, which indicates good agreement. Results for IHC $0 / 1+$ and $3+$ cases showed $100 \%$ concordance between FISH and DISH. All discordant cases belonged to the IHC 2+ category and details of the cases are shown in Table 4. It is interesting to note that three of these four discordant cases were in the equivocal category for either FISH or DISH.

\section{Discussion}

It is important to develop an accurate test for HER2 status in breast cancer so that patients can receive optimal treatment. A false-negative result may lead to delay or omission of HER2 targeting treatment. A false-positive one, however, may result in unnecessary treatment for the patient. This is particularly important because HER2 targeting drugs are known to cause rare but significant adverse effects, including serious cardiotoxicity. ${ }^{10}$ In addition, the cost of treatment is high and may be a financial burden for patients. 
(a)

(i) Bland-Altman plot for HER2 counts per nucleus. The mean difference (FISH-DISH) is 0.386 ; lowest line shows slightly higher bias with FISH, with greater discrepancy between FISH and DISH at

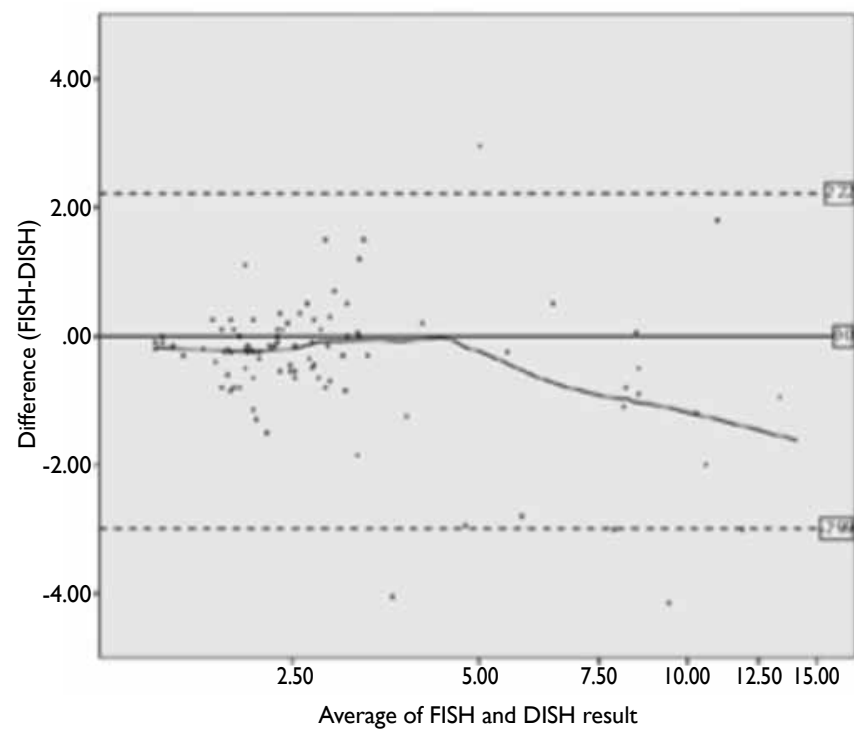

(b)

(i) Scatter plot for HER2 counts per nucleus for FISH and DISH. Dotted line is the line of equality (perfect concordance). Solid line represents linear regression, $y=0.965 x+0.506, R^{2}=0.804$, $\mathrm{P}<0.001$; Pearson's correlation coefficient $=0.897, \mathrm{P}<0.001$

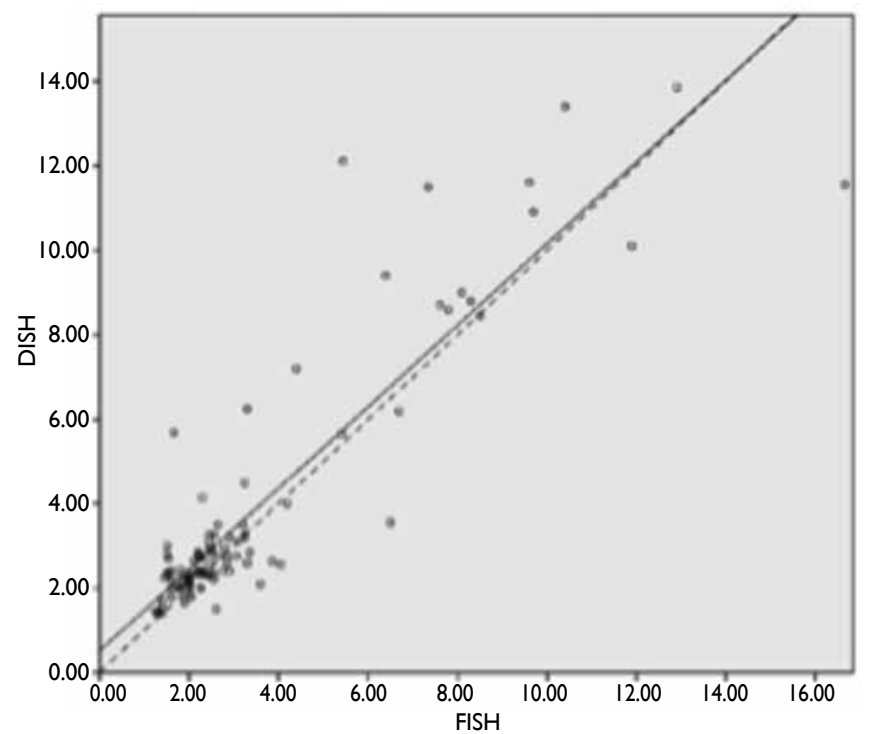
higher HER2 counts; $95 \% \mathrm{Cl},-2.99$ to 2.22 (dotted lines)

(ii) Bland-Altman plot for HER2/CEPI7 ratio. The mean difference (FISH-DISH) is 0.279 ; lowest line shows slightly higher bias with FISH for the majority for cases; $95 \% \mathrm{Cl},-0.87$ to 1.43 (dotted lines)

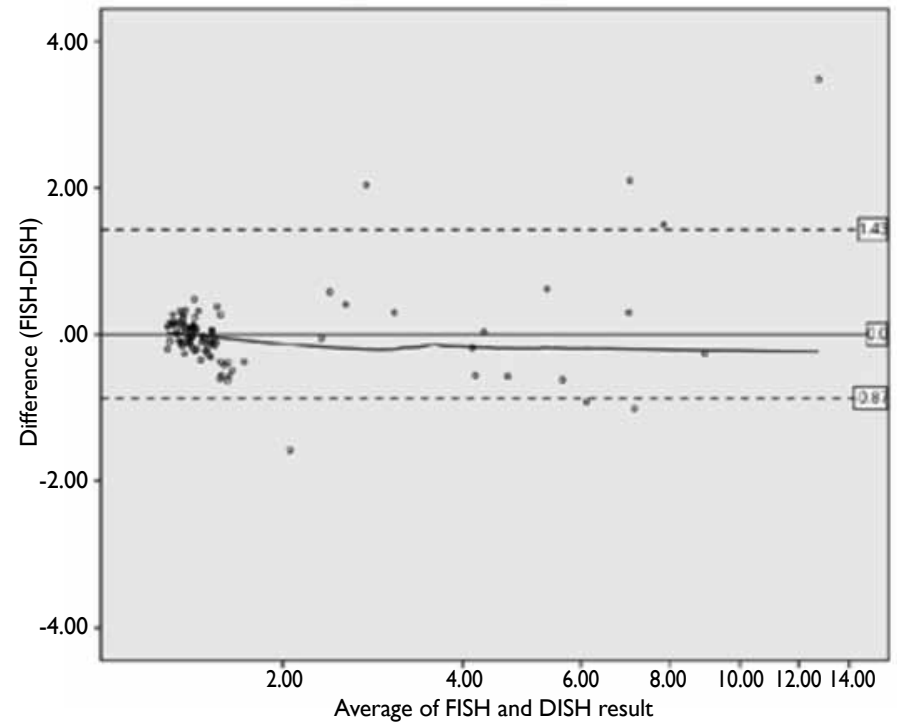

(ii) Scatter plot for HER2/CEPI7 ratio for FISH and DISH. Dotted line is the line of equality (perfect concordance). Solid line represents linear regression, and shows DISH results in lower estimates for HER2/CEPI7 ratios than FISH, $y=0.852 x+0.286, R^{2}=0.927$, $\mathrm{P}<0.00$ I; Pearson's correlation coefficient $=0.963, \mathrm{P}<0.00$ I

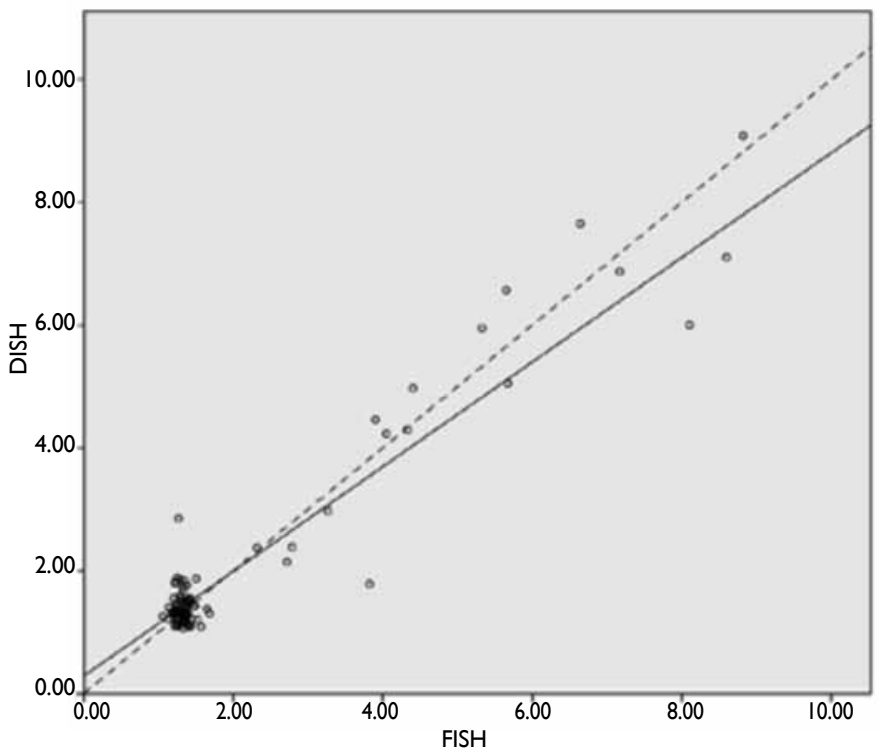

FIG 2. (a) Bland-Altman plots illustrating limits of agreement. The difference between each paired measurement (FISH-DISH) is plotted against the mean of the paired measurements. (b) Scatter diagrams illustrating correlation of DISH results with FISH results

Abbreviations: $\mathrm{Cl}$ = confidence interval; DISH = dual-colour in-situ hybridisation; FISH = fluorescence in-situ hybridisation

Various methods have been developed specificity, relatively low price, and short turnaround for HER2 testing. The ASCO/CAP guidelines time. Further, the immunostain does not degrade over recommend HER2 testing by IHC and ISH methods. time. Its sensitivity is variable, however, and affected Each has their own advantages and disadvantages. significantly by pre-analytic, analytic, and postThe advantages of IHC include its high analytic factors. ${ }^{2,11,22}$ Tissue fixation factors, such 
TABLE 4. The cases in which two assays showed non-concordance on amplification status

\begin{tabular}{|c|c|c|c|c|c|c|c|}
\hline \multirow[t]{2}{*}{ Case No. } & \multirow[t]{2}{*}{ IHC } & \multicolumn{3}{|c|}{ FISH } & \multicolumn{3}{|c|}{ DISH } \\
\hline & & $\begin{array}{c}\text { HER2/CEP17 } \\
\text { ratio }\end{array}$ & $\begin{array}{c}\text { HER2 per } \\
\text { nucleus }\end{array}$ & $\begin{array}{c}\text { Amplification } \\
\text { status }\end{array}$ & $\begin{array}{c}\text { HER2/CEP17 } \\
\text { ratio }\end{array}$ & $\begin{array}{c}\text { HER2 per } \\
\text { nucleus }\end{array}$ & $\begin{array}{c}\text { Amplification } \\
\text { status }\end{array}$ \\
\hline 8 & $2+$ & 1.65 & 4.05 & Equivocal & 1.38 & 2.55 & Non-amp \\
\hline 13 & $2+$ & 1.38 & 3.25 & Non-amp & 1.27 & 4.5 & Equivocal \\
\hline 26 & $2+$ & 1.35 & 2.30 & Non-amp & 1.84 & 4.15 & Equivocal \\
\hline 83 & $2+$ & 1.27 & 1.65 & Non-amp & 2.85 & 5.7 & Amplified \\
\hline
\end{tabular}

Abbreviations: DISH = dual-colour in-situ hybridisation; FISH = fluorescence in-situ hybridisation; IHC = immunohistochemistry; Non-amp $=$ non-amplified

as ethanol exposure and antigen retrieval methods, can lead to inaccurate IHC results. ${ }^{11}$ In an ideal setting, tissue for IHC should be fixed in $10 \%$ neutral buffered formalin for 6 to 48 hours, ${ }^{9}$ but in practice it is not uncommon for insufficient formalin to be used or for time-to-fixation to be often prolonged. ${ }^{11}$ There may also be scoring error. Although the use of controls can reduce interobserver variability, it cannot be eliminated. ${ }^{22}$

The general advantage of ISH methods compared with IHC is that ISH may be accurately performed on tissues fixed for variable lengths of time and in other fixatives. ${ }^{11}$ In addition, ISH can also be applied to a wide range of tissue samples, such as paraffin-embedded tissue, frozen samples, or micro-tissue arrays. ${ }^{2}$ Nonetheless, the different types of ISH also have their respective shortcomings.

The disadvantage for FISH is that, first, it is not possible to identify cell morphology and other histological features because it is visualised under fluorescence microscopy. Second, since the fluorescence of the probe will decay with time, samples cannot be archived. ${ }^{2}$ This makes it difficult for future retrieval and for re-examination. Third, sample preparation is complex and usually takes at least 2 days.

On the other hand, DISH makes use of brightfield microscopy that allows better delineation of cell morphology, tumour heterogeneity, and easier identification of tumour cells. ${ }^{2}$ Also, automation is possible so complexity and time required for sample preparation can be reduced. The DISH assay, however, is not perfect. One of its disadvantages is that analysis may sometimes fail. In our experience, the failure rate for DISH is somewhat higher than that for FISH. To date, there remain few studies published on the accuracy of DISH compared with FISH or IHC. ${ }^{17-20}$

This study provides more information about concordance of DISH and FISH, and is the first report from Hong Kong. In our study, FISH and DISH showed no statistical difference for HER2/CEP17 ratio and HER2 counts per nucleus. Correlation between the values was high. Pearson's correlation coefficient in our study was 0.963 for HER2/CEP17 ratio, and 0.897 for mean HER2 copies per nucleus. This is similar to the values reported by other studies, ranging from 0.79 to 0.81 by Gao et $\mathrm{a}^{17}$ and 0.85 to 0.87 by Horii et al. ${ }^{18}$ This indicates that DISH consistently correlates well with FISH for quantitative results. Our study also showed that FISH and DISH had a high level of agreement in classifying $H E R 2$ gene amplification status. BlandAltman plot showed good agreement between FISH and DISH. Agreement was less at a higher HER2/CEP17 ratio, and DISH tended to underestimate the result. This is similar to the findings by Mansfield et al..$^{20}$ Cohen's Kappa coefficient in our study was 0.882 . Reports by other studies vary, from $>0.9$ in the study by Horii et al ${ }^{18}$ in which only $48 \%$ of cases studied were of the equivocal IHC category, to 0.58 by Mansfield et $\mathrm{al}^{20}$ who focused on samples enriched for difficult-to-assess HER2 anomalies.

For our case series, the failure rate of FISH was $0.96 \%$. This is consistent with failure rates reported in the literature that range from $<1 \%$ to $8.4 \% .^{11,17,18,20}$ The failure rate for DISH in our case series was $3.85 \%$, which is slightly higher than the reported failure rate of $0 \%$ to $2.8 \%$ in previous studies. ${ }^{17,18,20}$ This may be explained by the fact that most cases in our series were of IHC 2+ category, which is the most challenging group of cases. It is worth noting that in all cases wherein FISH or DISH analysis failed, when one test failed, the other gave useful information on HER2 gene amplification status. Therefore the availability of both FISH and DISH assay allows one test to be used as an alternative, when the other fails.

The number of cases in our study was relatively small compared with other published studies, ${ }^{17,18,20}$ with only 83 consecutive cases of equivocal IHC cases within a given time period. Although it may be argued that the further 21 cases added in the IHC-positive or -negative category may constitute sampling bias, re-analysis of the data excluding these cases made no significant difference to the findings. Moreover, these 21 cases demonstrated 100\% concordance between FISH and DISH, supporting the robustness of both tests in straightforward cases. 
Indeed, of the four discordant cases between FISH and DISH, three were of the equivocal category by FISH or by DISH by the updated ASCO/CAP 2013 guidelines, but had given concordant non-amplified results both by FISH and by DISH using the earlier ASCO/CAP 2007 guidelines.

Another limitation of our study was that it was not prospective: only raw data of signal enumeration for FISH testing previously performed were available. The statistical analysis was based on reclassification of cases according to new ASCO/ CAP 2013 guidelines. Although the FISH and DISH slides were interpreted by different personnel, with the possibility of interobserver variability, the concordance between the two assays was very good.

\section{Conclusions}

Our study confirms that the determination of HER2 gene amplification status by DISH correlates well with that by FISH. In our laboratory, DISH would be a reliable and useful option for HER2 testing in breast cancer. Having both FISH and DISH assay available for service could help reduce the number of failed cases.

\section{Acknowledgements}

This study was supported by the SK Yee Medical Foundation (project number: 213218).

\section{References}

1. Cancer Registry 2011. Hospital Authority, Hong Kong. Available from: http://www3.ha.org.hk/cancereg/ rank_2011.pdf. Accessed Oct 2015.

2. Gutierrez C, Schiff R. HER2: biology, detection, and clinical implications. Arch Pathol Lab Med 2011;135:55-62.

3. Konecny G, Pauletti G, Pegram M, et al. Quantitative association between HER-2/neu and steroid hormone receptors in hormone receptor-positive primary breast cancer. J Natl Cancer Inst 2003;95:142-53.

4. Ménard S, Valagussa P, Pilotti S, et al. Response to cyclophosphamide, methotrexate, and fluorouracil in lymph node-positive breast cancer according to HER2 overexpression and other tumor biologic variables. J Clin Oncol 2001;19:329-35.

5. Joensuu H, Kellokumpu-Lehtinen PL, Bono P, et al. Adjuvant docetaxel or vinorelbine with or without trastuzumab for breast cancer. N Engl J Med 2006;354:809-20.

6. Piccart-Gebhart MJ, Procter M, Leyland-Jones B, et al. Trastuzumab after adjuvant chemotherapy in HER2positive breast cancer. N Engl J Med 2005;353:1659-72.

7. Slamon DJ, Leyland-Jones B, Shak S, et al. Use of chemotherapy plus a monoclonal antibody against HER2 for metastatic breast cancer that overexpresses HER2. N Engl J Med 2001;344:783-92.

8. Valero V, Forbes J, Pegram MD, et al. Multicenter phase III randomized trial comparing docetaxel and trastuzumab with docetaxel, carboplatin, and trastuzumab as first-line chemotherapy for patients with HER2-gene-amplified metastatic breast cancer (BCIRG 007 study): two highly active therapeutic regimens. J Clin Oncol 2011;29:14956.

9. Wolff AC, Hammond ME, Hicks DG, et al. Recommendations for human epidermal growth factor receptor 2 testing in breast cancer: American Society of Clinical Oncology/College of American Pathologists clinical practice guideline update. J Clin Oncol 2013;31:3997-4013.

10. Wolff AC, Hammond ME, Schwartz JN, et al. American Society of Clinical Oncology/College of American Pathologists guideline recommendations for human epidermal growth factor receptor 2 testing in breast cancer. J Clin Oncol 2007;25:118-45.

11. Sauter G, Lee J, Bartlett JM, Slamon DJ, Press MF. Guidelines for human epidermal growth factor receptor 2 testing: biologic and methodologic considerations. J Clin Oncol 2009;27:1323-33.

12. Yaziji H, Goldstein LC, Barry TS, et al. HER-2 testing in breast cancer using parallel tissue-based methods. JAMA 2004;291:1972-7.

13. Slamon DJ, Godolphin W, Jones LA, et al. Studies of the HER-2/neu proto-oncogene in human breast and ovarian cancer. Science 1989;244:707-12.

14. Slamon DJ, Clark GM, Wong SG, Levin WJ, Ullrich A, McGuire WL. Human breast cancer: correlation of relapse and survival with amplification of the HER-2/neu oncogene. Science 1987;235:177-82.

15. Owens MA, Horten BC, Da Silva MM. HER2 amplification ratios by fluorescence in situ hybridization and correlation with immunohistochemistry in a cohort of 6556 breast cancer tissues. Clin Breast Cancer 2004;5:63-9.

16. INFORM HER2 Dual ISH DNA Probe Cocktail-P100027. Available from: http://www.accessdata.fda.gov/cdrh_docs/ pdf10/P100027b.pdf. Accessed 6 Dec 2013.

17. Gao FF, Dabbs DJ, Cooper KL, Bhargava R. Brightfield HER2 dual in situ hybridization (DISH) assay vs fluorescence in situ hybridization (FISH): focused study of immunohistochemical 2+ cases. Am J Clin Pathol 2014;141:102-10.

18. Horii R, Matsuura M, Iwase T, Ito Y, Akiyama F. Comparison of dual-color in-situ hybridization and fluorescence in-situ hybridization in HER2 gene amplification in breast cancer. Breast Cancer 2014;21:598-604.

19. Koh YW, Lee HJ, Lee JW, Kang J, Gong G. Dual-color silverenhanced in situ hybridization for assessing HER2 gene amplification in breast cancer. Mod Pathol 2011;24:794800 .

20. Mansfield AS, Sukov WR, Eckel-Passow JE, et al. Comparison of fluorescence in situ hybridization (FISH) and dual-ISH (DISH) in the determination of HER2 status in breast cancer. Am J Clin Pathol 2013;139:144-50.

21. Yau TK, Sze H, Soong IS, Hioe F, Khoo US, Lee AW. HER2 overexpression of breast cancers in Hong Kong: prevalence and concordance between immunohistochemistry and in-situ hybridisation assays. Hong Kong Med J 2008;14:1305.

22. Perez EA, Cortés J, Gonzalez-Angulo AM, Bartlett JM. HER2 testing: current status and future directions. Cancer Treat Rev 2014;40:276-84. 